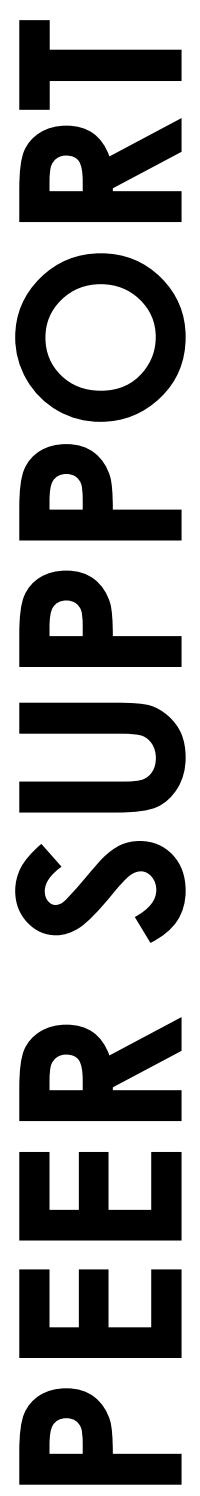

Keywords:

peer support, mental health, public health, online platform

\section{Mind Support: A Systematic Review of Online Peer Support Platforms}

\author{
Noah Stapper , Gema Benavides Jimenez", \\ Yasmin Barenco Abbas ${ }^{2}$, Maya Homsy King ${ }^{2}$ \\ ${ }^{1}$ University of Edinburgh, Scotland \\ ${ }^{2}$ University of Utrecht, The Netherlands
}

*Correspondence: noahstapper@yahoo.com

DOI: $\underline{\text { 10.31739/ANAMH.2021.1.21 }}$ 


\section{INTRODUCTION}

Health, as defined by the World Health Organization (WHO), is a state of "complete physical, mental and social well-being and not merely the absence of disease or infirmity". A state of mental health, therefore, is more than simply the absence of disorders or disabilities. This state of mental health, however, is disrupted for one in every three people at one point in their lifetime [1]. The WHO has reported that $76-85 \%$ of people with serious mental disorders living in developing countries, and 35$50 \%$ of those in developed countries do not receive treatment [2, 3]. The Department of Health in the UK predicted that $75 \%$ of people who want mental health treatment do not receive it [4] and a recent study estimates that only $16.5 \%$ of individuals suffering from major depressive disorder receive adequate treatment [5]. Difficulties with mental health are one of the main causes of the overall disease burden worldwide [6] and predictions for the future are dire. It is projected that by 2025, the US will have shortages "in nearly every type of mental health care provider (e.g., psychiatrist, psychologist, social worker)" [5].

Currently there is a global shortage of mental health care providers, including psychiatrist, psychologists and mental health nurses [7, 8]. Therefore, a preventative approach has been proposed to combat the difficulties at an earlier stage and therefore reduce the needed care [8]. Additional support systems are needed that do not rely on the scarce resource of mental health care providers. One part of this solution is to invest in the growth of peer support interventions that offer acute and accessible mental health support for everyone, do not rely on mental health care providers and are especially effective for mild cases of mental health difficulties and therefore prevent the emergence of more severe cases.

Mind Support [9] is a non-profit organization whose product is an online platform to combat the mental health crisis using free, individualized peer support. In the following article we will explain the concept of the nonprofit foundation Mind Support corporation (German eingetragener Verein (e.V.)) and will then systematically review the current literature that supports and underlies the concept of online peer support.

\section{Mind Support foundation}

Mind Support is a non-profit foundation that offers a free online platform. Users can anonymously get in touch with someone else who has previously experienced the, or similar mental health difficulties they are currently experiencing. Users take an online interactive questionnaire that assesses their complaints and the causes of their complaints, and are then matched, by means of an automatic algorithm, with a user who has had similar experiences. The match is made by assessing similarities in reported complaints of both users, who take the same questionnaire. The algorithm matches users to supporters with the highest compatibility based on the questionnaire. Supporters must be at least $55 \%$ compatible to be matched with a user. However, if a user is matched with a peer who they feel is incapable of helping them, or that they feel incapable of helping, they can ask to switch, and will be re-matched. This will allow for refining the match between users and supporters and guarantee that the match is made not only based on similarity of experiences but also the needs of the user, ensuring a more fitting match.

Before entering into the chat room, users will be presented with a message that encourages them to leave the conversation if they feel uncomfortable, to not ask for identifying details, be respectful at all times and to focus on listening and sharing, rather than giving direct advice. This is necessary, as the conversation will be anonymous and unsupervised. This feature should support the advantages of online peer support, such as anonymity reducing stigma and perception of differences between users [10].

Mind Support does not aim to offer therapy; it is there to support individuals with subclinical complaints and to reassure users that other people have overcome the same, or similar difficulties that they are experiencing. Mind Support is an example of an organization that could assist in filling the gap created by the public mental health services, as it provides people who have more mild mental health complaints with a place for support.

The Mind Support platform will benefit its users by way of the advantages addressed in this paper and attempt to decrease the concerns of online peer-support platforms through features such as a report button, safety notice before entering a chat, and a precautionary distinction between those with mild and serious mental health complaints.

A distinction is made by categorising any complaints below the threshold of diagnosis in the DSM-V, as "mild". This is the target audience, yet more severe cases are also attended to since Mind Support can also be an accessible addition to therapy for those who suffer from more severe difficulties, or support for individuals on the waiting list for professional therapy. Any users presenting risk of harm to themselves or others do not progress into the chat function and instead are shown an emergency notice. 
Further, as justified below, certain categories will be excluded from the "mutual support" option.

\section{PEER SUPPORT}

\section{What is peer support}

In the context of mental health, peer support is the process in which we recognise our shared experiences of disadvantage and distress and come together to support and learn from each other while making an interpersonal connection [11]. Research has highlighted the importance of both giving and receiving support when defining peer support. This suggests that this reciprocity is what increases the sense of mutual support, trust and empathy within peer support relationships [12, 13].

Why is peer support effective?

Although the popularity of peer support continues to grow, few studies have examined the exact mechanism of how peer support works, and it is still poorly understood. In 2003, a study by Dennis [14] described three overlapping mechanisms for possible beneficial effects of peer support. According to her, peer support interventions may have a direct effect by decreasing isolation and increasing sharing of health and selfmanagement information, a buffering effect by reducing the impact of stressors, and a mediating effect by providing positive role modelling.

A recent study by Watson [13] describes the use of lived experience as the defining feature of peer support. In particular, the honest sharing of emotions such as anger and guilt in relation to particular experiences enables peers to build connections and to feel "normal", as others had experienced similar events as well [15]. The study speculates that one of the reasons why the lived experience mechanism by which peer support acts is so powerful in these types of relationships are the traditional cultures of non-disclosure within mental health services. These cultures increase the saliency of the sharing, while creating a contrast to the overt hierarchies and clinical understandings which often define mental health services. Hence, this is one example of how the powerful cultures of mental health systems contribute to the uniqueness of peer support, and of how the culture of peer support might offer alternatives and positive influences to conventional practice and contribute to cultural change [13].

\section{Is online peer support effective?}

With the rise of social media and the disruption of normal life due to COVID-19, there has been a prominent switch from physical to online peer support groups. Online peer support comes with advantages and disadvantages. Starting with the latter, online peer support might exclude some people due to inability to access the internet or illiteracy [16]. Furthermore, there is a risk for the appearance of inaccurate, dangerous or mistaken medical information [17].

When comparing in-person to online support however, there are many benefits associated with the latter. First, in contrast with in-person groups which are restricted by time, space and geographic boundaries, online support can be available anywhere and at any time. Due to the larger pool of people who can use the platform, it is easier to find someone with similar struggles. There also seems to be a gender difference in the use of online compared to in-person support groups. Research suggests that men use online support more frequently than in-person peer support groups compared to women [18], while women are more likely to have been professionally treated for mental health problems [4]. Online support groups have the potential to reach population groups which previously may have been difficult to reach by other public health intervention programs. The previously mentioned benefits of online support groups coupled with the anonymity that this service provides may encourage men to participate online more freely, especially when talking about sensitive issues like depression or suicide [10]. Moreover, while inperson support groups tend to be associated with more costly diagnoses and expensive treatments, online support favours conditions that are poorly understood, difficult or impossible to cure, or are overlooked by traditional health care [19]. Lastly, as a result of the absence of sociodemographic or visual cues, online groups tend to be more egalitarian and less concerned with social status. People with disabilities or stigmatizing diseases can participate without concern that they will be prejudged. Hence, online groups often serve to muster advocacy and political action to change the status quo [16].

Online peer support as a mechanism of early intervention in mental health

The US Surgeon General said "Children and families are suffering because of missed opportunities for prevention and early identification, fragmented services, and low priorities for resources" [20]. This is relevant for physical health and for mental health as well. In 2014, a study by Clark et al. in New Zealand revealed that approximately 
one in every five adolescents will suffer from a recognized mental health disorder each year in most developed nations [21, 22]. Approximately three-quarters of adults with psychiatric disorders present symptoms before the age of 18 years [23]. Therefore, the screening and early identification and treatment of mental health disorders during adolescence may aid in reducing mental health issues among vulnerable populations, as this is key in preventing development of the disorder to a more severe stage [24].

Indeed, there is growing evidence that shows early intervention during adolescence can be associated with reduced severity of symptoms and improved adult mental health outcomes [24, 25]. A stronger focus on prevention and early intervention programs would contribute to detecting and treating mild cases at an early stage. Thus, development into more serious and distressing mental health problems could be prevented.

Online peer support platforms such as Mind Support offer a space for individuals with subclinical complaints to address their problems and therefore demonstrate a preventative tool towards the maturation of these problems into larger disorders. Many people do not search for help until a later and more severe stage in their trajectory of mental health difficulties, due to the mental health stigma. Mind Support aims to normalise the presence of subclinical mental health complaints in the majority of the population by advocating for people with subclinical complaints to sign up. People may then feel more comfortable and confident to address their problems at an earlier stage.

\section{Disadvantages of online peer support}

\section{Online safety and peer contagion}

The social contagion theory or peer contagion theory encompasses the belief that negative emotional states can spread through groups of intimate social networks similar to the spreading of infectious diseases [26]. In other words, could the communication between two people during the process of peer support worsen their mental health states? Online peer support platforms fight the potential threats of peer contagion by connecting users who have previously experienced the mental health difficulties with someone who is currently experiencing these. This decreases the risk of the person receiving support being negatively influenced by the supporter, since the supporter is no longer experiencing the same negative emotions. Yet the risk of relapse for the person supporting does persist. Therefore, it is important that the supporter is stable and that they are reminded to exit the conversation when "old feelings" reoccur. In this case the health of the supporter is more important than the possible negative side effects of the abrupt ending of the conversation. The supportee will be able to connect to a new supporter in this case.

In an article written by Bastiampillai and colleagues (2013), the conclusion is drawn that peer contagion is a process that could potentially occur, yet it could cause the occurrence of mood disorders or could also be a potential solution or treatment for mood disorders, since positive moods can also be contagious [26].

Many platforms, including Mind Support e.V. include mutual support. Mutual support connects two people who are experiencing complaints at the present moment supporting each other. This combination is most susceptible to the dangers of peer contagion. Therefore, additional safety measures are taken for this option. Eating disorders and adolescents are excluded from this support option and additional safety notices are given to the user. People dealing with anorexia [27] and adolescents [28] are excluded since previous literature has shown that these groups are most susceptible to peer contagion.

Many articles that thematise peer contagion or social contagion investigate the prevalence of peer contagion in social groups, but there is very little literature that focuses on peer contagion specifically in peer support settings. Online peer support is anonymous, which changes the social forces usually at play, and is between two people at different stages of their recovery; therefore, positive peer contagion seems intuitively more likely. However, more literature is needed to assess the potential dangers and benefits (positive peer contagion) of peer contagion for online peer support platforms.

Unsupervised online chat rooms come with many risks. One article written by Webb et al. [29] analysed the risks of unsupervised online chat rooms for adolescents. The article demonstrated two main risks; firstly, there is the possibility that adults contact adolescents with the aim of taking advantage of their vulnerability. Secondly, there may be contagion with other members who are "ruminating about feeling depressed" [29], making suicide pacts or describing suicide or self-harming intentions.

\section{Advantages of online peer support}

\section{Peer support as a way to support those forgotten by the public mental health system}

In the past couple of years, the potential of peer support to inspire hope, strengthen and enable the individual to cope better with their mental health [11] and support their recovery, has started to be recognised by a number of 
mental health services providers in the U.S., Australia, New Zealand and Europe [13].

The way that mental health services are typically structured is as a series of steps, each of these embodying notions of clinical severity and mental capacity and every step up or down is potentially a source of disruption to people's lives (Gillard, 2019). In this illness-focused approach, connections to the community, family and friends are negatively impacted over time and isolation is imposed on people for whom isolation may be already at the root of their distress. The distinctiveness of peer support lies in its trauma-informed approach, which focuses on what people have experienced before in their lives beyond the disorder or difficulty, rather than what might be wrong with them as an individual [30]. This social intervention gives the opportunity for creating and normalizing non-treatment-based relationships [15], and through these relationships, peer support works to strengthen connections to the community [31].

Additionally, we are becoming increasingly aware that peer support can play an important role in addressing persistent inequity in access to mental health care for marginalised groups within society [12], as well as a resource in Lower and Middle Income Countries (LMICs). Peer support has the potential to address the 'treatment gap', which refers to the difference between the number of people who need care, and those who actually receive it. This gap is especially significant in LMICs [32].

\section{Effectiveness of low threshold online support options}

Over the past few centuries, the mental health interventions designed to treat psychological disorders have considerably improved, yet the burden of mental health remains high [33]. Moreover, research has highlighted the substantial need in human resources, suggesting models that include task shifting and nonprofessional-assisted interventions [34]. Despite the associated positive outcomes of non-professional assisted intervention [35], there are a few limitations such as the direct costs associated with training, employment and connecting with non-professionals. Nevertheless, these difficulties have been minimized through the increased use of technology, which can provide methods to screen, train and connect non-professionals with those who need their support [36]. In fact, since technology makes it much easier for people to volunteer, train and engage with one another, technology-assisted interventions have the potential to increase the human resources available for providing emotional support. It has also been demonstrated that people in emotional distress find the support offered through online platforms to be helpful. Additionally, online support has the power to provide a feasible way to better connect the communities and to promote well-being and altruistic actions among the population [36].

A more recent study from Stawarz et al. [37] reported that smartphone apps were the most commonly used technology by the public to support their mental health and well-being. However, even though the use of digital platforms results in a feasible intervention in terms of cost and availability [38], technology should not be seen as a replacement for traditional psychotherapy. Rather, it offers new opportunities to support mental health as part of an overall ecosystem of mental health services.

\section{Peer support as a supplement to professionally-lead and medical treatment}

While antidepressant medication is deemed an effective treatment for major depressive disorder, a large multicenter effectiveness study by Rush et al. [39] demonstrated that among patients who achieve remission with antidepressants, more than half relapse within one year. This suggests that additional services are needed to help patients cope with continued symptoms even while they receive current evidence-based treatments such as medication. According to the U.S. government health commission, peer support services have many attractive features to recommend them as a supplement to standard depression treatment, and to become essential components of recovery-based mental health care.

As a matter of fact, a study by Yalom [40] described peer support as having many similar features to more conventional practices of group psychotherapy, such as altruism, cohesiveness, universality, imitative behaviour, instillation of hope, and catharsis. Therefore, in light of previous evidence, a study by Pfeiffer et al., [41] aimed to assess whether peer support interventions resulted in greater reduction of depressive symptoms compared to either usual care or psychotherapy. Results pooled from randomized controlled trials indicate that peer support interventions improve depression symptoms more than usual care alone and that the effects may be comparable to those of group cognitive behavioural therapy. Hence, these findings suggest that peer support interventions have the potential to be effective components of depression care and provide overall evidence in support of expert opinion advocating for peer support to be included in recovery-oriented mental health treatment.

Moreover, a study by Baumel [36], revealed that while the advantages of psychotherapy revolved around experience and professional ability to diagnose and provide the right reflection of their patient's difficulties, the advantages of peer support were tied to the sincere care and support 
users received. Besides, the users suggested that receiving support from unpaid volunteers made them feel that the support was more genuine. Despite the benefits of psychotherapy by a professional, which has unique advantages such as decreased risks and diagnosis, peer support provides a certain level of comfort and therapeutic gain that cannot be replicated by professional psychotherapists, since they are paid and do not tend to relate to their patients' difficulties from the perspective of a peer.

A study by Baumel and Schueller in 2016 [42], aimed to investigate the effects and evaluations of patients using an online support platform to supplement treatment for women with perinatal depression and anxiety. The study found that patients evaluated the use of the platform as useful and their attitudes toward the trained listeners were positive. Overall, patients noted a need for support outside the scheduled therapy time and believed that freely available online emotional support could help meet this need. They also reported enjoying receiving support from those who suffered in the past from perinatal mood disorders. Hence, this study highlights the effectiveness as well of introducing accessible and available online support into existing treatment for a diverse range of mental health problems.

\section{Barriers in seeking mental health services for young adults}

Nevertheless, despite the evident benefits of treating mild concerns early, it seems that little is known about the referral and management of adolescents with mild to moderate mental health concerns in primary care and community settings [43]. Some of the most common identified barriers include the lack of opportunistic screening for mental health concerns among young people in primary care settings and the lack of recognition of mental health problems [44]. There are also a variety of individual factors that serve as barriers for young people trying to access mental health services. These barriers include the young person's perception that there is nothing wrong, or that nothing will help [45], and a lack of knowledge about where to access help [46]. Lastly, structural barriers, including socioeconomic barriers, have also been identified by a number of studies as a significant factor in reducing access to mental health care [47], along with perceptions of discrimination by health professionals [48].

\section{CONCLUSION}

In conclusion, peer support relationships are based on reciprocal sharing of similar mental health difficulties to learn and support each other. Online platforms such as Mind Support provide an easily accessible and anonymous tool to connect on the basis of shared lived experiences. This has increased the potential to reach groups which previously may have been difficult to reach by the public health intervention programs.

Online peer support demonstrates two main risks; the vulnerability of adolescents in online chat rooms and the risk of peer contagion. Mind Support e.V does take additional measures to minimize these risks, yet in some cases they may persist.

Online peer support platforms such as Mind Support e.V. have the potential to inspire hope and strengthen as well as to enable individuals to cope better with their mental health. The identified need for prevention and for intervention in mental health, has highlighted that the distinctiveness of peer support relationships compared to other mental health services could offer new opportunities to support mental health as part of an overall ecosystem of mental health services.

\section{REFERENCES}

1. Steel, Z., C. Marnane, C. Iranpour, T. Chey, J.W. Jackson, V. Patel, and D. Silove, The global prevalence of common mental disorders: a systematic review and meta-analysis 1980-2013. International journal of epidemiology, 2014. 43(2): p. $476-493$.

2. Demyttenaere, K., R. Bruffaerts, J. PosadaVilla, I. Gasquet, V. Kovess, J. Lepine, M.C. Angermeyer, S. Bernert, G. de Girolamo, and P. Morosini, Prevalence, severity, and unmet need for treatment of mental disorders in the World Health 
Organization World Mental Health Surveys. Jama, 2004. 291(21): p. 25812590.

3. $\quad$ Edwards, J., I. Goldie, I. Elliott, J. Breedvelt, L. Chakkalackal, and U. Foye, Fundamental facts about mental health 2016. Mental health foundation, 2016.

4. Singleton, N. and G. Lewis, Better Or Worse: A Longitudinal Study of the Mental Health of Adults Living in Private Households in Great Britain: Report Based on Surveys Carried Out by the Office for National Statistics in 2000 and 2001for the Department of Health and the Scottish Executive Health Department. Stationery Office, 2003.

5. Rudd, B.N. and R.S. Beidas, Digital mental health: the answer to the global mental health crisis.? Jmir Mental Health, 2020. 7(6): p. e18472.

6. Vos, T., R.M. Barber, B. Bell, A. BertozziVilla, S. Biryukov, I. Bolliger, F. Charlson, A. Davis, L. Degenhardt, and D. Dicker, Global, regional, and national incidence, prevalence, and years lived with disability for 301 acute and chronic diseases and injuries in 188 countries, 1990-2013: a systematic analysis for the Global Burden of Disease Study 2013. The Lancet, 2015. 386(9995): p. 743-800.

7. Butryn, T., L. Bryant, C. Marchionni, and F. Sholevar, The shortage of psychiatrists and other mental health providers: causes, current state, and potential solutions. International Journal of Academic Medicine, 2017. 3(1): p. 5 .

8. Wainberg, M.L., P. Scorza, J.M. Shultz, L. Helpman, J.J. Mootz, K.A. Johnson, Y. Neria, J.-M.E. Bradford, M.A. Oquendo, and M.R. Arbuckle, Challenges and opportunities in global mental health: a research-to-practice perspective. Current psychiatry reports, 2017. 19(5): p. 28.

9. $\quad$ Mind Support e. V. 2021 [cited 2021; Available from: www.mindsupport.eu.

10. Salem, D.A., G.A. Bogat, and C. Reid, Mutual help goes on-line. Journal of Community Psychology, 1997. 25(2): p. 189-207.

11. Gillard, S., Peer support in mental health services: where is the research taking us, and do we want to go there? 2019, Taylor \& Francis.

12. Faulkner, A. and J. Kalathil, The freedom to be, the chance to dream: Preserving user-led peer support in mental health. London: Together for Mental Wellbeing, 2012.

13. Watson, E., The mechanisms underpinning peer support: a literature review. Journal of Mental Health, 2017.
14. Dennis, C.-L., Peer support within a health care context: a concept analysis.

International journal of nursing studies, 2003. 40(3): p. 321-332.

15. Gidugu, V., E.S. Rogers, S. Harrington, M. Maru, G. Johnson, J. Cohee, and J. Hinkel, Individual peer support: A qualitative study of mechanisms of its effectiveness.

Community mental health journal, 2015. 51(4): p. 445-452.

16. White, M. and S.M. Dorman, Receiving social support online: implications for health education. Health education research, 2001. 16(6): p. 693-707.

17. Dickerson, S.S., D.M. Flaig, and M.C. Kennedy, Therapeutic connection: help seeking on the Internet for persons with implantable cardioverter defibrillators. Heart \& Lung, 2000. 29(4): p. 248-255.

18. Klemm, P. and M.T. Nolan. Internet cancer support groups: legal and ethical issues for nurse researchers. in Oncology Nursing Forum. 1998.

19. Davidson, L. and K. Guy, Peer support among persons with severe mental illnesses: a review of evidence and experience. World psychiatry, 2012. 11(2): p. 123-128.

20. General, U.S.P.H.S.O.o.t.S., U.S.O.o.D. Prevention, H. Promotion, C.f.D. Control, and Prevention, The Surgeon General's call to action to prevent and decrease overweight and obesity. 2001: US Government Printing Office.

21. Costello, E.J., S. Mustillo, A. Erkanli, G. Keeler, and A. Angold, Prevalence and development of psychiatric disorders in childhood and adolescence. Archives of general psychiatry, 2003. 60(8): p. 837-844.

22. Patel, V., A.J. Flisher, S. Hetrick, and P. McGorry, Mental health of young people: a global public-health challenge. The Lancet, 2007. 369(9569): p. 1302-1313.

23. Fergusson, D.M., L.J. Horwood, E.M. Ridder, and A.L. Beautrais, Subthreshold depression in adolescence and mental health outcomes in adulthood. Archives of general psychiatry, 2005. 62(1): p. 66-72.

24. Shochet, I.M. and R. Hoge, Resourceful adolescent program: A prevention and early intervention program for teenage depression. 2009.

25. Chanen, A.M., H.J. Jackson, L.K. McCutcheon, M. Jovev, P. Dudgeon, H.P. Yuen, D. Germano, H. Nistico, E. McDougall, and C. Weinstein, Early intervention for adolescents with borderline personality disorder: quasi-experimental comparison with treatment as usual. Australian \& New Zealand Journal of Psychiatry, 2009. 43(5): p. 397-408. 
26. Bastiampillai, T., S. Allison, and S. Chan, Is depression contagious? The importance of social networks and the implications of contagion theory. Australian \& New Zealand Journal of Psychiatry, 2013. 47(4): p. 299-303.

27. Allison, S., M. Warin, and T. Bastiampillai, Anorexia nervosa and social contagion: Clinical implications. Australian \& New Zealand Journal of Psychiatry, 2014. 48(2): p. 116-120.

28. Stevens, E.A. and M.J. Prinstein, Peer contagion of depressogenic attributional styles among adolescents: A longitudinal study. Journal of Abnormal Child Psychology, 2005. 33(1): p. 25-37.

29. Webb, M., J. Burns, and P. Collin, Providing online support for young people with mental health difficulties: challenges and opportunities explored. Early intervention in psychiatry, 2008. 2(2): p. 108-113.

30. Blanch, A., B. Filson, D. Penney, and C. Cave, Engaging women in trauma-informed peer support: A guidebook. Alexandria, VA: National Center for Trauma-Informed Care, 2012.

31. Gillard, S., S. Gibson, J. Holley, and M. Lucock, Developing a change model for peer worker interventions in mental health services: a qualitative research study. Epidemiology and Psychiatric Sciences, 2015. 24(5): p. 435.

32. Puschner, B., Peer support and global mental health. Epidemiology and Psychiatric Sciences, 2018. 27(5): p. 413414.

33. Collins, P.Y., V. Patel, S.S. Joestl, D. March, T.R. Insel, A.S. Daar, I.A. Bordin, E.J. Costello, M. Durkin, and C. Fairburn, Grand challenges in global mental health. Nature, 2011. 475(7354): p. 27-30.

34. Kazdin, A.E. and S.M. Rabbitt, Novel models for delivering mental health services and reducing the burdens of mental illness. Clinical Psychological Science, 2013. 1(2): p. 170-191.

35. Chowdhary, N., S. Sikander, N. Atif, N. Singh, I. Ahmad, D.C. Fuhr, A. Rahman, and V. Patel, The content and delivery of psychological interventions for perinatal depression by non-specialist health workers in low and middle income countries: a systematic review. Best practice \& research Clinical obstetrics \& gynaecology, 2014. 28(1): p. 113-133.

36. Baumel, A., Online emotional support delivered by trained volunteers: users' satisfaction and their perception of the service compared to psychotherapy.
Journal of mental health, 2015. 24(5): p. 313-320.

37. Stawarz, K., C. Preist, and D. Coyle, Use of smartphone apps, social media, and webbased resources to support mental health and well-being: Online survey. JMIR mental health, 2019. 6(7): p. e12546.

38. Baumel, A., C.U. Correll, and M. Birnbaum, Adaptation of a peer based online emotional support program as an adjunct to treatment for people with schizophrenia-spectrum disorders. Internet interventions, 2016. 4: p. 35-42.

39. Rush, A.J., M.H. Trivedi, S.R. Wisniewski, A.A. Nierenberg, J.W. Stewart, D. Warden, G. Niederehe, M.E. Thase, P.W. Lavori, and B.D. Lebowitz, Acute and longer-term outcomes in depressed outpatients requiring one or several treatment steps: a $S T A R$ * D report. American Journal of Psychiatry, 2006. 163(11): p. 1905-1917.

40. Yalom, I. and M. Leszcz, The Theory and Practice of Group Psychotherapy. 5th Edn Basic Books. New York, NY, 2005.

41. Pfeiffer, P.N., M. Heisler, J.D. Piette, M.A. Rogers, and M. Valenstein, Efficacy of peer support interventions for depression: a meta-analysis. General hospital psychiatry, 2011. 33(1): p. 29-36.

42. Baumel, A. and S.M. Schueller, Adjusting an available online peer support platform in a program to supplement the treatment of perinatal depression and anxiety. JMIR mental health, 2016. 3(1): p. e5335.

43. Clark, T.C., E.A. Johnson, M. Kekus, J. Newman, P.S. Patel, T. Fleming, and E. Robinson, Facilitating Access to Effective and Appropriate Care for Youth With Mild to Moderate Mental Health Concerns in New Z ealand. Journal of Child and Adolescent Psychiatric Nursing, 2014. 27(4): p. 190-200.

44. Johnson, J.G., E.S. Harris, R.L. Spitzer, and J.B. Williams, The patient health questionnaire for adolescents: validation of an instrument for the assessment of mental disorders among adolescent primary care patients. Journal of Adolescent Health, 2002. 30(3): p. 196-204.

45. Vanheusden, K., C.L. Mulder, J. van der Ende, F.J. van Lenthe, J.P. Mackenbach, and F.C. Verhulst, Young adults face major barriers to seeking help from mental health services. Patient education and counseling, 2008. 73(1): p. 97-104.

46. Coggan, C., P. Patterson, and J. Fill, Suicide: Qualitative data from focus group interviews with youth. Social science \& medicine, 1997. 45(10): p. 1563-1570. 
47. Sareen, J., A. Jagdeo, B.J. Cox, I. Clara, M. ten Have, S.-L. Belik, R. de Graaf, and M.B. Stein, Perceived barriers to mental health service utilization in the United States, Ontario, and the Netherlands. Psychiatric services, 2007. 58(3): p. 357364.
48. Crengle, S., E. Robinson, S. Ameratunga, T. Clark, and D. Raphael, Ethnic discrimination prevalence and associations with health outcomes: data from a nationally representative cross-sectional survey of secondary school students in New Zealand. BMC public health, 2012. 12(1): p. 1-11. 